\title{
Gastric Dysplasia-Like Epithelial Atypia Associated with Chemoradiotherapy for Esophageal Cancer: A Clinicopathologic and Immunohistochemical Study of 15 Cases
}

\author{
Thomas P. Brien, M.D., Francis A. Farraye, M.D., Robert D. Odze, M.D., F.R.C.P.(c) \\ Departments of Pathology (TPB, RDO) and Gastroenterology (FAF), Brigham and Women's Hospital and \\ Harvard Medical School, Boston, Massachusetts
}

Preoperative chemotherapy combined with radiotherapy (chemrad) is a common type of neoadjuvant treatment for esophageal adenocarcinoma or squamous cell carcinoma. The purpose of this study was to describe the clinical, histologic, proliferative (MIB-1), and oncogenetic (p53) features of 15 patients with gastric dysplasia-like epithelial atypical changes associated with preoperative chemrad for esophageal cancer. Two of these cases were initially misinterpreted as dysplasia, which led to partial gastrectomy. The findings were compared with 12 age- and sex-matched patients with known gastric dysplasia. Cases with gastric dysplasia-like epithelial atypia were significantly associated with a flat gross appearance, a patchy distribution, foveolar and gland involvement, surface maturation, an open nuclear chromatin pattern with prominent nucleoli, retention of nuclear polarity, mitoses confined to the pits, lack of atypical mitoses, cytoplasmic hypereosinophila and/or vacuolization, a lack of association with intestinal metaplasia, and finally, irregular glandular microcystic change, in comparison to the dysplasia controls. Furthermore, the study cases showed MIB-1 positivity restricted to the deep foveolar epithelium and an absence of p53 staining in 14 of 15 cases, in contrast to the dysplasia controls, in which MIB-1 stained both the deep and superficial foveolar epithelium and surface epithelium, and p53 was positive in all cases (100\%). In summary, a number of histologic and immunohistochemical features may distinguish gastric dysplasia-like epithelial atypia associated with chemrad for esophageal cancer from true dys-

Copyright () 2001 by The United States and Canadian Academy of Pathology, Inc.

VOL. 14, NO. 5, P. 389, 2001 Printed in the U.S.A.

Date of acceptance: December 22, 2000

Address reprint requests to: Robert D. Odze, M.D., F.R.C.P.(c), Department of Pathology, Brigham and Women's Hospital, 75 Francis Street, Boston, MA 02115; e-mail: rdodze@bics.bwh.harvard.edu; fax: 617-277-9015. plasia. Pathologists should be aware of this entity and its histologic and immunohistochemical features to avoid misinterpretation and prevent unnecessary treatment.

KEY WORDS: Atypia, Chemotherapy, Dysplasia, Esophageal cancer.

Mod Pathol 2001;14(5):389-396

Preoperative chemotherapy combined with radiotherapy (chemrad) is an increasingly popular method of neoadjuvant treatment for esophageal adenocarcinoma and squamous cell carcinoma. It remains controversial whether there is a clear survival advantage with the use of chemrad (1-5). However, recent prospective studies have shown increased survival after chemrad if given before surgical resection (4-6). Thus, many institutions currently use neoadjuvant chemrad, followed by surgical resection, as a primary treatment for patients with Stage II or III esophageal cancer.

Anecdotal observations by the authors of this manuscript have noted that severe epithelial atypical changes that mimic dysplasia, or even adenocarcinoma, may occur in the stomach of esophageal cancer patients who have received preoperative chemrad. In several instances, the atypical changes were initially misinterpreted as being neoplastic, which, in retrospect, led to unnecessary treatment such as partial gastrectomy. Interestingly, in the majority of cases, the esophagus showed severe chemrad-associated esophagitis with ulceration but did not contain atypical changes in the residual Barrett's (columnar) epithelium when present.

Therefore, the purpose of this study was to evaluate the clinical, histologic, proliferative, and oncogenetic features of 15 patients with gastric dysplasia-like epithelial atypical changes associated with preoperative chemrad for esophageal cancer 
and to compare them with 12 known gastric dysplasia specimens as controls.

\section{MATERIALS AND METHODS}

\section{Study Group}

Twelve esophageal cancer patients with gastric dysplasia-like epithelial atypical changes in their postchemrad esophagogastrectomy specimens were identified from a review of 160 (7.5\%) consecutive patients with esophageal cancer who were treated with chemrad before their resection at the Brigham and Women's Hospital in Boston, MA between the years 1992 and 1999. The surgical specimens from another 120 patients with esophageal cancer who did not receive preoperative chemrad during this same time period were also reviewed for gastric atypia, but none were found from this cohort. An additional three cases were identified from one of the authors' (RDO) personal consult service. Of the 15 cases, 2 were interpreted by the original pathologist as intramucosal adenocarcinoma, 6 as dysplasia (4 low grade, 2 high grade), 4 as indefinite for dysplasia, and 3 as probably reactive. Informed consent was obtained from all patients or their guardians. Of the 15 patients, 13 had adenocarcinoma, and 2 had squamous cell carcinoma. All patients received preoperative chemrad. The protocol consisted of two cycles of 5-fluorouracil and cisplatinum given concurrently with radiation therapy (total dose, 50.4 Gy given in 1.8-Gy fractions for $51 / 2$ weeks). All patients were then treated surgically by a radical en bloc esophageal (and partial stomach) resection, with lymph node dissection, with an intent to cure. Clinical follow-up, including the results of subsequent endoscopic examinations, was obtained. In one instance, an autopsy was performed 4 weeks postoperatively.

As controls, 12 biopsy or resection specimens from 7 patients with definite gastric dysplasia (9 low grade and 3 high grade) were retrieved from the pathology files of the Brigham and Women's Hospital for comparison with the study cases. These controls were age and sex matched with the study cases.

\section{Pathologic Evaluation}

Routinely processed hematoxylin and eosinstained sections from the gastric portion of the esophagogastrectomy specimens and either the mucosal biopsy or resection specimen of the dysplasia control cases were evaluated for a variety of histologic features. These included the location (antrum, fundus, cardia) and distribution (focal, multifocal, diffuse) of the atypical changes, its gross appearance (flat or polypoid), presence of surface maturation, the degree of nuclear pseudostratification, loss of polarity, hyperchromaticity, pleomorphism and chromatin quality (vesicular or clumped), appearance of nucleoli (number, prominent, inconspicuous), nuclear/cytoplasmic (N/C) ratio (low, normal, or increased), presence and location of mitoses and atypical mitoses, and the quality of cytoplasmic mucin (depleted, bubbly, eosinophilic). The features in the adjacent gastric mucosa (intestinal metaplasia, degree and location of inflammation, presence of gland atrophy) were also evaluated.

The esophageal portion of the specimens was evaluated with two sections of the proximal margin, at least six representative sections of tumor, when present, and two sections of the tumor/mucosa interface. A thorough lymph node sampling was also performed. For cases in which tumor was not identified grossly (because of positive response to preoperative chemrad), the entire ulcerated area of the esophagus was submitted for histological examination. In all cases, at least five sections of the surrounding Barrett's epithelium, when present, were evaluated.

\section{Immunohistochemical Evaluation}

Immunohistochemical studies for MIB-1 (proliferation marker) and p53 (tumor suppresser gene product) were performed using the standard avidin-biotin complex technique. These antibodies were chosen for analysis because gastric dysplasia often shows surface epithelium MIB-1 positivity, indicating aberrant localization of proliferative cells, and p53 mutations (7). Four-micrometer-thick tissue sections were prepared from Forman-fixed, paraffin-embedded tissue blocks. After baking for one hour at $60^{\circ} \mathrm{C}$, the sections were deparaffinized and rinsed for 5 minutes under running water. Endogenous peroxidase activity was blocked by incubating the slides in $3 \% \mathrm{H}_{2} \mathrm{O}_{2}$ in methanol for 5 minutes. The slides were then microwave heated in an $800-\mathrm{W}$ microwave oven at $199^{\circ} \mathrm{F}$ for 30 minutes in $10 \mathrm{~mm}$ citrate buffer at $\mathrm{pH}$ 6.0. After cooling for 15 minutes, the slides were transferred to phosphate buffered saline (Fisher Scientific, Agawam, Massachusetts) and then blocked with $1.5 \%$ horse serum (Vector Laboratories Inc., Burlingame, California) for 15 minutes. The slides were then incubated with monoclonal mouse anti-human antibody to either MIB-1 (clone MIB-1, 1:200 dilution, AMAC Inc/ Immunotech, Westbrook, Maine) or p53 (clone DO-1, 1:50 dilution, Oncogene Research Products/Calbiochem, Cambridge, Massachusetts) for 1 hour in a humid chamber at room temperature. After a 5-minute wash in phosphate buffered saline, the secondary antibody (Vectastain Elite ABC kit, Mouse IgG, Vector Laboratories) was applied according to the manufacturer's instructions. The sections were developed using 3,3'-diaminobenzidine (3,3'-diaminobenzidine) (Sigma Chemical Company, St. Louis, Mis- 
souri) as substrate and counterstained with Gill's hematoxylin (Fisher Scientific). Lymph node tissue was used as a positive control for p53, and tonsil tissue was used for MIB-1. Lymphocytes within the lamina propria also served as an internal positive control for MIB-1.

Nuclei that stained with an intensity equal to or greater than the controls were considered positive. The distribution of positive staining within the foveolae, glands, and surface epithelium was recorded. All slides were read by two of the authors (TPB and RDO) with minimal $(<1 \%)$ interobserver variability, which, when present, was resolved at a multiheaded microscope.

\section{Statistics}

The proportion of cases with each feature was compared between the study and control groups, and the differences were statistically analyzed using the $\chi^{2}$ model (STATA version 5.0).

\section{RESULTS}

\section{Clinical Features and Follow-up Data}

The male/female $(\mathrm{M} / \mathrm{F})$ ratio of the study patients was $6.5: 1$, and their mean age was 59 years (range:
30 to 79 years). The M:F ratio of the controls was 5.0:1, and their mean age was 60 years (range: 59 to 78 years). Clinical follow-up information was available in 10 of the 15 study patients. Eight of $10(80 \%)$ patients had died of their disease (esophageal cancer) at the time of this study (mean survival: 28 months). The causes of death were as follows: two from postoperative complications, five from metastatic disease, and one from locally recurrent carcinoma. One patient had an autopsy 4 weeks after resection, and no abnormal changes were noted in the stomach. One of the deceased patients had a normal upper endoscopic examination (esophagus and stomach) 4 months after surgery. The two patients who were still alive both had recent endoscopic examinations (4 and 12 months after resection, respectively) with no endoscopic evidence of gastric dysplasia or dysplasia-like changes in their gastric mucosal biopsies.

\section{Pathologic Features}

A summary of the pathologic features and a comparison with the dysplasia controls are presented in Table 1. Thirteen patients had Barrett's esophagusassociated adenocarcinoma (seven Stage II, six Stage III), and two had squamous cell carcinoma

\section{TABLE 1. Summary of the Pathologic Features of the Study Cases and Controls}

\begin{tabular}{|c|c|c|c|}
\hline Features & Study Cases, $n=15$ (\%) & Dysplasia Controls, $n=12(\%)$ & $P$ Value \\
\hline \multicolumn{4}{|l|}{ Anatomic distribution } \\
\hline Flat appearance & $15(100)$ & $8(67)$ & $=.02$ \\
\hline Foveolar and glandular atypia & $14(93)$ & $0(0)$ & $<.001$ \\
\hline Glandular atypia only & $1(7)$ & $0(0)$ & NS \\
\hline Foveolar atypia only & $0(0)$ & $12(100)$ & $<.001$ \\
\hline Patchy distribution & $14(93)$ & $1(8)$ & $<.001$ \\
\hline Surface maturation & $7(50)$ & $0(0)$ & $<.001$ \\
\hline \multicolumn{4}{|l|}{ Nuclear features } \\
\hline Nuclear pseudostratification & $12(80)$ & $12(100)$ & NS \\
\hline Hyperchromasia & $14(93)$ & $12(100)$ & NS \\
\hline Enlarged nuclei & $14(93)$ & $12(100)$ & NS \\
\hline Pleomorphic nuclei & $7(47)$ & $8(67)$ & NS \\
\hline Clumped chromatin & $3(21)$ & $11(92)$ & $<.001$ \\
\hline Prominent nucleoli & $6(43)$ & $2(17)$ & $<.05$ \\
\hline Increased nuclear/cytoplasmic ratio & $4(27)$ & $8(67)$ & NS \\
\hline Loss of polarity & $0(0)$ & $8(67)$ & $<.001$ \\
\hline Multinucleation & $0(0)$ & $0(0)$ & NS \\
\hline \multicolumn{4}{|l|}{ Mitoses } \\
\hline Frequent mitoses ${ }^{\mathrm{a}}$ & $9(60)$ & $11(92)$ & NS \\
\hline Mitoses in upper foveolae or surface epithelium & $0(0)$ & $8(67)$ & $<.001$ \\
\hline Atypical mitoses & $1(7)$ & $5(42)$ & $=.04$ \\
\hline \multicolumn{4}{|l|}{ Cytoplasmic features } \\
\hline Mucin depletion & $14(93)$ & $12(100)$ & NS \\
\hline Hypereosinophilia & $13(87)$ & $0(0)$ & $<.001$ \\
\hline Vacuolization (glands only) & $7(47)$ & $0(0)$ & $<.001$ \\
\hline \multicolumn{4}{|l|}{ Glandular features } \\
\hline Atrophy & $7(47)$ & $8(67)$ & NS \\
\hline Irregular microcystic change & $11(73)$ & $0(0)$ & $<.001$ \\
\hline \multicolumn{4}{|l|}{ Adjacent gastric mucosa features } \\
\hline Intestinal metaplasia & $2(13)$ & $12(100)$ & $<.001$ \\
\hline Increased mononuclear inflammation only & $6(40)$ & $4(33)$ & NS \\
\hline Increased mononuclear and neutrophilic inflammation & $3(20)$ & $1(8)$ & NS \\
\hline
\end{tabular}

NS, not significant.

${ }^{\text {a }}$ Frequent mitoses $\geq 4$ mitoses/foveolae. 
(both Stage III). Of the 15 patients, 10 had no residual tumor in their resection specimen, three had microscopic residual tumor, and two had macroscopic residual tumor.

The prevalence of gastric dysplasia-like epithelial atypia in our esophageal cancer population was $7.5 \%$ ( $12 / 160$ cases). Of the 15 cases, all had atypical changes in the corpus. Grossly, the atypical areas were inconspicuous, showing no distinction from the surrounding mucosa. Histologically, atypical changes were present in the foveolar and glandular epithelium in 14 (93\%) and in the glandular epithelium only in one (7\%) case (Fig. 1). Epithelial atypia involved foveolae in a discontinuous, or patchy, fashion (Fig. 1). Affected pits were separated by areas of normal-appearing epithelium. Foveolar atypia consisted of slightly elongated pits containing pseudostratified cells with mucin depletion, hypereosinophilic cytoplasm, normal or slightly low N/C ratio, hyperchromatic oval to pencil-shaped enlarged nuclei with a nonclumped (open) chromatin pattern, and prominent nucleoli, either single or multiple (Fig. 2). Rare cases (3) showed a clumped chromatin pattern. Furthermore, in some cases, cytoplasmic tufting was present, particularly in the upper parts of the foveolar epithelium. Mitoses were frequent (one to four per foveola) but not atypical, except for one case. Mitoses were always confined to the lower two thirds of the pit epithelium. Seven cases (50\%) showed evidence of surface maturation characterized by a decrease in the degree of nuclear pseudostratification and increased cytoplasmic mucin production, whereas the remainder of cases showed atypical changes involving the upper foveolar and surface epithelium similar to dysplasia. Severe atypical epithelial changes were not seen in the residual esophageal columnar epithelium in any of the 13 cases with Barrett's esophagus-associated adenocarcinoma.

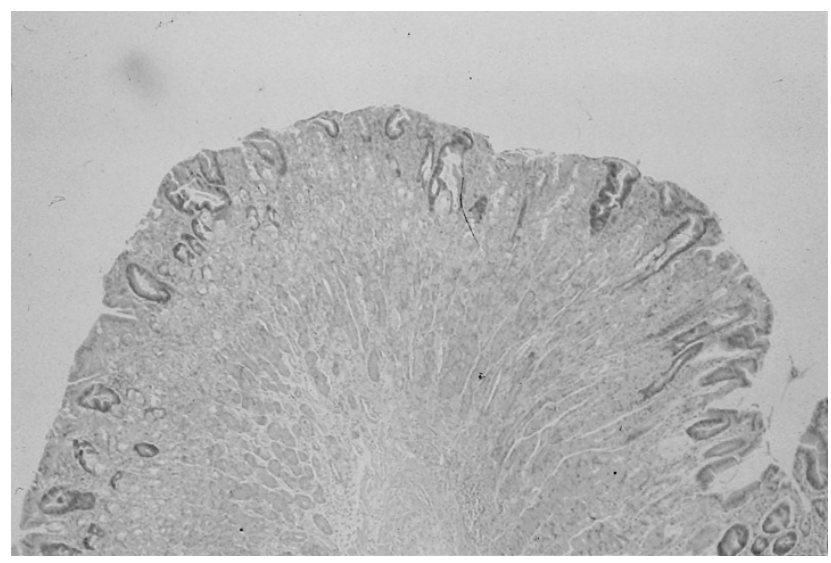

FIGURE 1. Low-power view of the gastric corpus showing discontinuous, or patchy, foveolar atypia. In between areas of epithelial atypia, there is a mild degree of mononuclear inflammation in the superficial lamina propria.
In contrast to the foveolar epithelium, affected glands revealed atrophic features, such as irregular microcystic change lined by attenuated, or flattened, parietal and chief cells (Fig. 3). The lumen of the microcysts occasionally showed neutrophilic and cellular debris (three cases). The cells lining the microcysts contained elongated and bubbly cytoplasm with enlarged round-to-oval-shaped nuclei showing an open chromatin pattern and inconspicuous, or small, eccentrically located nucleoli. Some cells in the glandular epithelium showed hypereosinophilic cytoplasm similar to that seen in the foveolar epithelium. However, similar to the foveolar epithelium, the N/C ratio of affected glandular cells was either normal or low, and abnormal mitoses were not present. In two cases, the glandular changes caused diagnostic confusion with adenocarcinoma by the consulting pathologist.

The unaffected gastric mucosa showed ulceration in three cases, all of which revealed dysplasia-like atypical epithelial changes adjacent to the ulcer bed. Increased inflammation (mononuclear in six cases, mononuclear and neutrophilic in three cases) was often present in the superficial mucosa. Intestinal metaplasia was focally present in $2(13 \%)$ cases. Table 1 summarizes some of the histologic features in the study cases in comparison to the dysplasia controls. A number of features were significantly more common in the study cases, such as a flat gross appearance, a patchy distribution, lack of association with intestinal metaplasia, foveolar and gland involvement, surface maturation, open nonclumped nuclear chromatin pattern with prominent nucleoli, retention of nuclear polarity, mitoses confined to the pits, lack of atypical mitoses, cytoplasmic hypereosinophilia, and/or vacuolization, and finally, irregular glandular microcystic change.

\section{Immunohistochemistry Results}

In all study patients, MIB-1 nuclear positivity was confined to the deep foveolar region (Fig. 4A). There was no staining in the upper foveolar or surface epithelium. In contrast, all dysplasia controls showed full-length foveolar and surface epithelial nuclear staining for MIB-1 (Fig. 4B).

p53 nuclear staining was present in only 1 of 15 study patients, and in this case, it was weak, focal, and limited to the deep foveolar epithelium (Fig. 5A). In contrast, all the dysplasia controls showed diffuse, strong, full-thickness foveolar and surface epithelial staining for p53 (Fig. 5B). The differences in MIB-1 and p53 staining between the study and the control group were statistically significant $(P<$ $.001)$. 

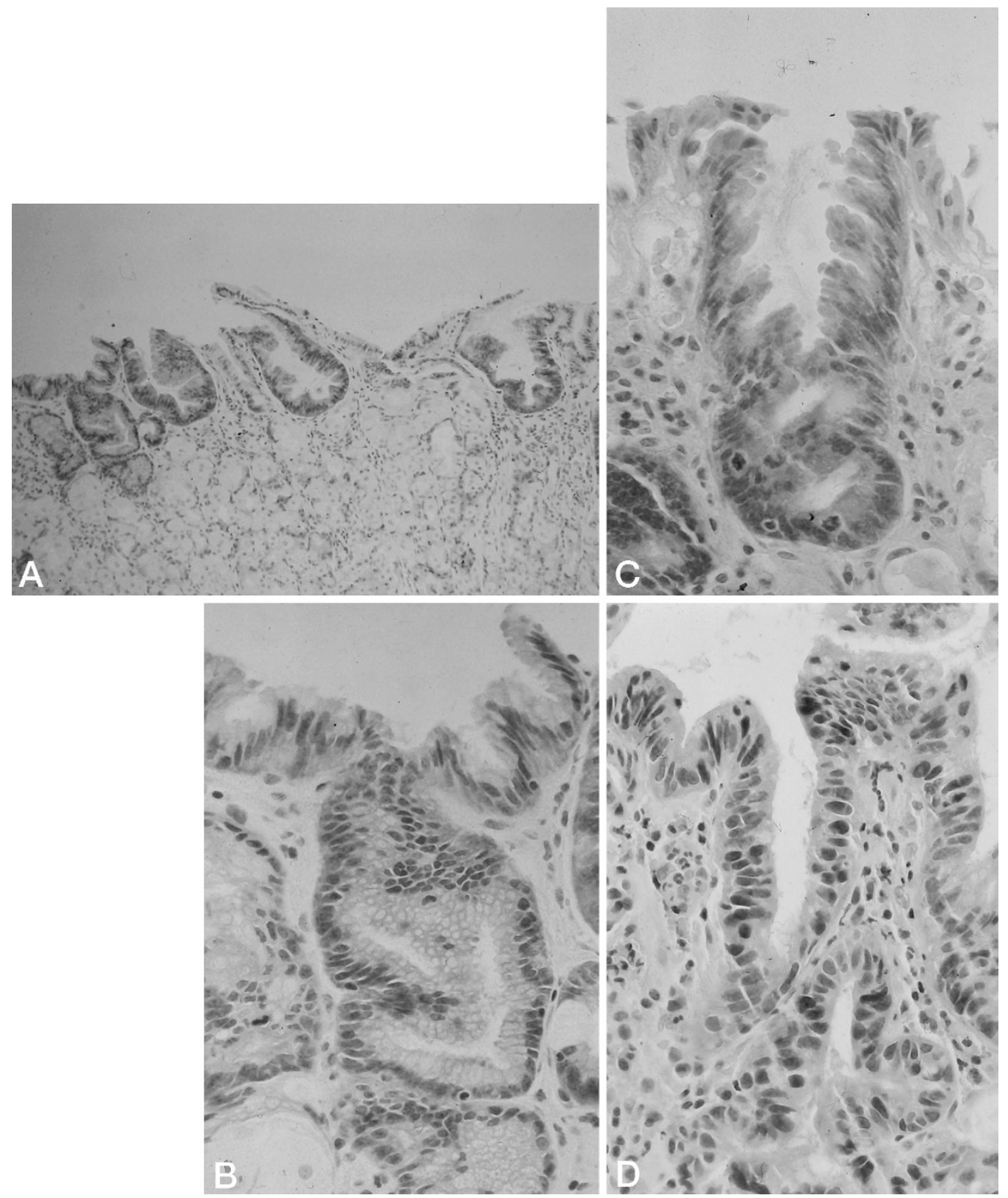

FIGURE 2. (A) Medium-power view of an area of gastric foveolar atypia showing slightly elongated and irregular pits containing pseudostratified, mucin-depleted cells. Some of the pits have a serrated appearance. (B) High-power view from another area of the same case showing cells with regular round-to-oval-shaped nuclei and prominent nucleoli. No atypical mitoses, significant pleomorphism, or loss of polarity is present. (C) Highpower view of another case showing marked cytoplasmic hypereosinophilia and cytoplasmic tufting. The nuclei are oval or slightly elongated and have a clumped chromatin pattern. Note the presence of mitoses in the lower half of the atypical epithelium. (D) For comparison, this is an example of high-grade gastric dysplasia. In contrast to our study cases, this epithelium contains cells with more pleomorphism, loss of polarity, and lack of surface maturation. Focal goblet cell metaplasia is present as well.

\section{DISCUSSION}

In this study, we described the histologic and immunohistochemical features of 15 patients with gastric dysplasia-like epithelial atypia secondary to chemrad for esophageal cancer. The prevalence of these changes in our chemrad-treated esophageal cancer population was $7.5 \%$. Dysplasia-like gastric atypia was only found in chemrad-treated patients. We did not find these changes in a review of 120 esophageal cancer resections in patients who did not receive preoperative chemrad. Thus, we are confident that these changes were, in fact, related to chemrad.

Compared with the dysplasia controls, our study patients showed a significantly higher proportion of cases with a flat gross appearance, a patchy distribution, lack of association with intestinal metaplasia, foveolar and gland involvement, surface maturation, open nonclumped nuclear chromatin pattern with prominent nucleoli, retention of nuclear polarity, mitoses confined to the pits, lack of atypical mitoses, cytoplasmic hypereosinophilia 




FIGURE 3. (A) Medium-power view of the base of the mucosa from a case with foveolar and glandular atypia. Some of the glands show an irregular microcystic configuration. The cells lining the glands have a flattened appearance. Note the presence of inflammatory debris in some of the glandular lumens. (B) High-power view of a gland microcyst containing flattened atypical cells with hyperchromatic, enlarged, and slightly irregular nuclei. This degree of atypia may be mistaken for an infiltrating adenocarcinoma. However, note the normal, or low, nuclear/cytoplasmic ratio of these cells. Also, the cytoplasm of some of the cells is slightly vacuolated.

and/or vacuolization, and finally, irregular glandular microcystic change. Thus, gastric dysplasia-like epithelial changes can be distinguished from true gastric dysplasia by careful attention to these histologic features. In addition, we also found that immunohistochemistry for MIB-1 or p53 may be helpful. Specifically, true dysplasia showed fullthickness foveolar and surface MIB-1 and p53 staining, whereas the study cases showed only deep foveolar MIB-1 staining and only rare focal p53 staining. These results are not surprising because p53 abnormalities are frequently observed in gastric carcinoma as well as in its dysplastic precursor (7). This data, combined with the fact that no atypia was noted in the few patients who had follow-up tissue available for review, supports our belief that these changes probably represent a marked repair reaction to chemrad.

Of particular interest is that the atypical changes noted in the stomach of our patients were not observed in the residual esophageal columnar epithe-
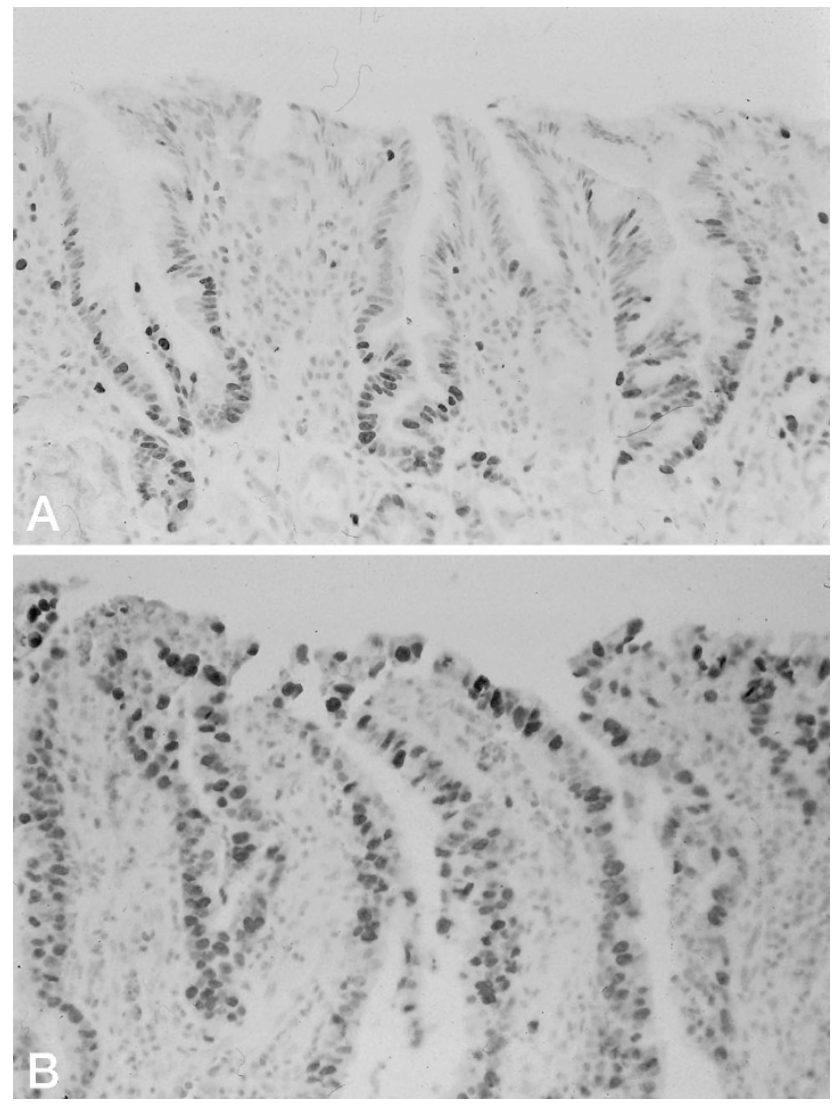

FIGURE 4. (A) High-power view of an area of gastric foveolar atypia stained with MIB-1. Note that the majority of MIB-1 staining is confined to the lower two thirds of the pits, with little or no staining in the upper portion and surface of the epithelium. (B) In contrast to Panel A, this high-power view of a high-grade gastric dysplasia case shows full-thickness MIB-1 staining, including the upper portions of the foveolar epithelium and the surface epithelium.

lium of the 13 patients who had Barrett's esophagus-associated adenocarcinoma. However, of the 13 patients, only five had residual Barrett's epithelium remaining in their resection specimen. The rest of the cases showed severe, presumably chemrad related, ulceration of the esophagus. The reasons why the epithelium of the stomach and not the esophagus showed severe dysplasia-like atypia are unclear. One reason may be that the stomach was located at the periphery of the primary radiation field and, thus, may have been subjected to a lower dose, perhaps not enough to cause cell death but enough to cause injury followed by repair. Another reason may be related to an inherent disparity in the sensitivity of these two organs to the adverse affects of chemrad. For instance, the esophagus is known to be more tolerant to the principal effects of radiation in comparison to the stomach $(8-10)$. However, this data applies to squamous epithelium and not to metaplastic columnar epithelium characteristic of Barrett's esophagus.

The chemotherapy agents used in our study patients were 5-flurouracil and cisplatinum. 5 -flurouracil is a pyridine analogue that acts as an 


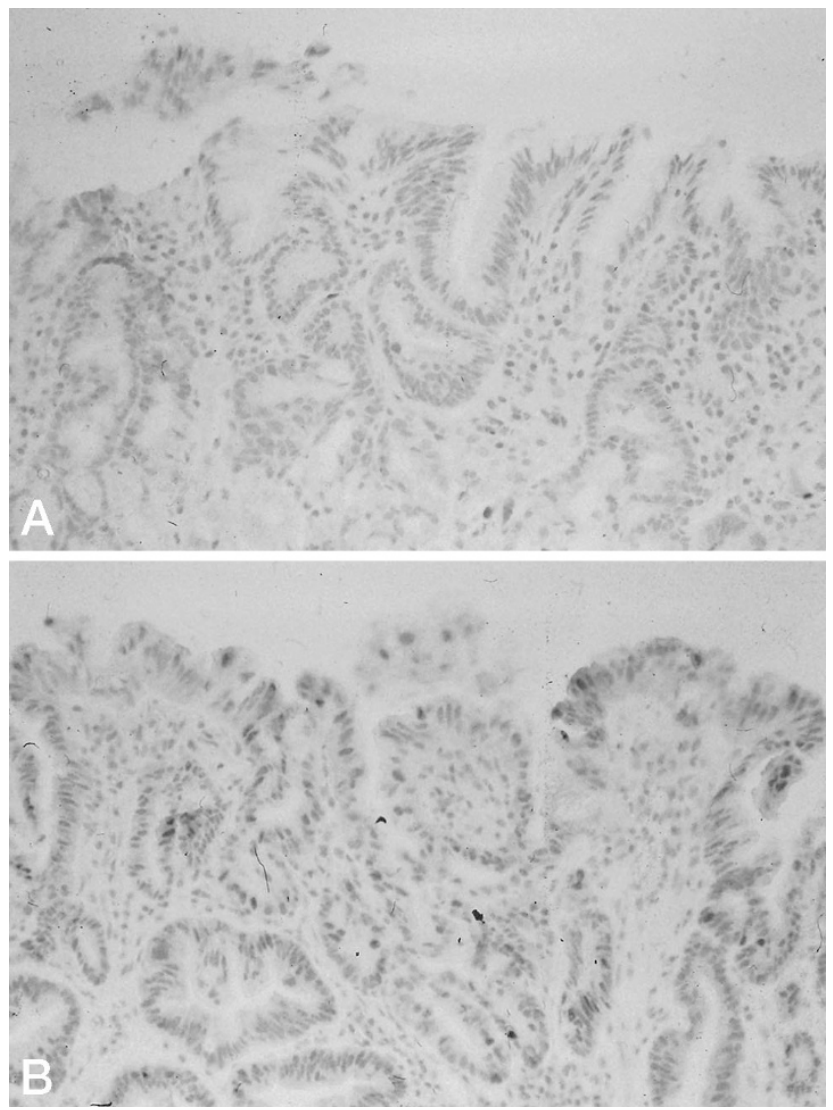

FIGURE 5. (A) Medium-power view of an area of gastric foveolar atypia stained with p53 showing complete absence of staining in the atypical epithelium. (B) Another case of gastric high-grade dysplasia stained with p53 showing strong, full-thickness nuclear staining for p53.

antimetabolite to uracil (11). As a result, it interferes with the synthesis of DNA by blocking the conversion of deoxyuridylic acid to thymidylic acid (11, 12). Cisplatinum is a platinum-containing complex that acts in a similar manner to other alkylating agents (13). Selective killing of tumor cells is probably due to attack on the guanine- and cytosinerich regions of DNA and the formation of platinum crosslinks between DNA strands (13). It is believed that the actions of these drugs produce damage that is potentially repairable by normal cells. However, because of their damaging effects on DNA, one can speculate that regenerating human cells may go through a phase of genotypic and phenotypic atypia in their pathway to repair.

External-beam radiation may also cause tissue injury, followed by an atypical repair reaction, as a consequence of its effect on nuclear DNA $(10,14-$ 18). In fact, these effects, such as enlargement of cell nuclei, may be potentiated by the concurrent use of chemotherapy and are typically long-lasting (9). Interestingly, chief and parietal cells have been noted to be more sensitive than mitotically active foveolar neck cells (16) and may help explain the atypia seen in the gastric glands in the cases from this study.
Neoadjuvant, or adjuvant, chemotherapy has been used in the treatment of other types of gastrointestinal malignancies as well. In fact, atypical epithelial changes have been observed in some instances (19-26). For example, hepatic arterial infusion chemotherapy has been reported as an effective method of treating both primary and metastatic colonic carcinoma of the liver $(27,28)$. Interestingly, there have been several previously published reports of gastric and duodenal ulceration, with epithelial atypia, following this form of chemotherapy (19-22, 24-26). The epithelial atypia described in some of these cases was marked and, similar to some of our cases, was initially interpreted as carcinoma (26). In another instance, severe atypia suggestive of dysplasia completely normalized after ulcer therapy with sucralfate and cimetidine (21).

In another study by Petras et al. (24) of seven cases of gastric ulcer after hepatic arterial infusion chemotherapy for metastatic colon cancer, the following histologic features were found to be indicative of chemotherapy-associated atypia rather than carcinoma: preservation of mucosal architecture, atypia accentuated in the basilar gastric glands, bizarre atypia with cellular enlargement exceeding that seen in carcinoma, preservation of a low N/C ratio, cytoplasmic eosinophilia with vacuolization, few or no mitotic figures, cytologic resemblance to radiation effect, similar atypia within fibroblasts and endothelial cells, and an absence of intestinal metaplasia in the adjacent gastric epithelium. Some of these features are similar to the ones identified in our study cases. One difference of note is that we did not see bizarre atypia in our cases. However, our study used different chemotherapeutic agents and a systemic route of administration, which may account for the differences.

Epithelial atypia has also been described in cytology specimens after chemotherapy $(19,20,23)$. Several studies have reported bizarre atypia in gastric brushings after hepatic arterial infusion chemotherapy $(19,20)$. In one of these studies (3), the atypia was severe enough to cause an erroneous cytologic interpretation of malignancy (20). A study of esophageal-brush specimens from patients without esophageal or gastric cancer who had received various chemotherapeutic agents showed moderate to severe epithelial atypia mimicking cancer in 3 of 10 cases (23). Cytologic atypia, after chemotherapy, that mimics malignancy has been reported in the conjunctiva (29) and sinonasal tract (30) as well.

In summary, in this study we describe the histologic and immunohistochemical features of 15 patients with gastric dysplasia-like epithelial atypia secondary to chemrad for esophageal cancer. The prevalence of these atypical changes in our study population was $7.5 \%$. Pathologists should be aware 
of this entity, and its histologic and immunohistochemical features as described in this manuscript, to avoid misinterpretation and prevent unnecessary treatment.

\section{REFERENCES}

1. Kelsen DP, Ginsberg R, Quin L, Gunderson L, Mortimer J, Estes $\mathrm{N}$, et al. Chemotherapy followed by operation versus operation alone in the treatment of patient with localized esophageal cancer: a preliminary report of intergroup study. Proc Am Soc Clin Oncol 1997;16:227A.

2. Naunheim KS, Petruska PJ, Roy TS, Schlueter JM, Kim H, Baue AE. Multimodality therapy for adenocarcinoma of the esophagus. Ann Thorac Surg 1995;59:1085-91.

3. Torres C, Turner JR, Wang HH, Richards W, Sugarbaker D, Shahsafaei A, et al. Pathologic prognostic factors in Barrett'sassociated adenocarcinoma. Cancer 1999;85:520-8.

4. Urba S, Orringer M, Turrisi, Whyte R, Iannettoni M, Forastiere A. A randomized trial comparing surgery $(\mathrm{S})$ to preoperative concommitant chemoradiation plus surgery in patients (pts) with resectable esophageal cancer (CA): updated analysis. Proc Am Soc Clin Oncol 1992;10:1037-43.

5. Walsh TN, Noonan N, Hollywood D, Kelly A, Keeling N, Hennessy TPJ. A comparison of multimodal therapy and surgery for esophageal adenocarcinoma. N Engl J Med 1996; 335:462-7.

6. Minsky BD. Carcinoma of the esophagus. Part 2: adjuvant therapy. Oncology 1999;13:1415-27.

7. Stemmermann G, Heffelfinger SC, Noffsinger A, Hui YZ, Miller MA, Fenoglio-Preiser CM. The molecular biology of esophageal and gastric cancer and their precursors: oncogenes, tumor suppression genes, and growth factors. Hum Pathol 1994;25:968-81.

8. Bloomer WD, Hellman S. Normal tissue responses to radiation therapy. N Engl J Med 1975;293:80-3.

9. Sher ME, Bauer J. Radiation-induced enteropathy. Am J Gastroenterol 1990;85:121-8.

10. Novak JM, Collins JT, Donowitz M, Farman J, Sheahan DG, Spiro HM. Effects of radiation on the human gastrointestinal tract. J Clin Gastroenterol 1979;1:9-39.

11. Au JL-S, Rustum YM, Ledesma EJ, Mittelman A, Creaven PJ. Clinical pharmacological studies of concurrent infusion of 5-fluorouracil and thymidine in treatment of colorectal carcinomas. Cancer Res 1982;42:2930-7.

12. Casper ES. Phase 1 and clinical pharmacological evaluation of biochemical modulation of 5-fluorouracit with $N$-(phosphonacetyl)-L-aspartic acid. Cancer Res 1983;43:2324-9.

13. Sadler PJ. Inorganic pharmacology. Chem Br 1982;18:182-8.

14. Chowhan NM. Injurious effects of radiation on the esophagus. Am J Gastroenterol 1990;85:115-20.
15. Vanagunas A, Jacob P, Olinger E. Radiation-induced esophageal injury: a spectrum from esophagitis to cancer. Am J Gastroenterol 1990;85:808-12.

16. Clayman CB, Palmer WL, Kirsner JB. Gastric irradiation in the treatment of peptic ulcer. Gastroenterology 1968;55:403.

17. Berthrong M, Fajardo LF. Radiation injury in surgical pathology. II. Alimentary tract. Am J Surg Pathol 1981;5:153-78.

18. Fajardo LF, Berthrong M. Radiation injury in surgical pathology. Part I. Am J Surg Pathol 1978;2:159-99.

19. Becker SN, Sass MA, Petras RE, Hart WR. Bizarre atypia in gastric brushings associated with hepatic arterial infusion chemotherapy. Acta Cytol 1986;30:347-50.

20. Choi HY, Takeda M. Gastric epithelial atypia following hepatic arterial infusion chemotherapy. Diagn Cytopathol 1985;1:241-4.

21. Jewell LD, Fields AL, Murray CJ, Thomson AB. Erosive gastroduodenitis with marked epithelial atypia after hepatic arterial infusion chemotherapy. Am J Gastroenterol 1985;80: 421-4.

22. Kwee WS, Wils JA, Schlangen J, Nuyens CM, Arends JW. Gastric epithelial atypia complicating hepatic arterial infusion. Histopathology 1994;24:151-4.

23. O'Morchoe PJ, Lee DC, Kozak CA. Esophageal cytology in patients receiving cytotoxic drug therapy. Acta Cytol 1983; 27:630-4.

24. Petras RE, Hart WR, Bukowski RM. Gastric epithelial atypia associated with hepatic arterial infusion chemotherapy. Its distinction from early gastric carcinoma. Cancer 1985;56: 745-50.

25. Schuger L, Peretz T, Goldin E, Durst AL, Okon E. Duodenal epithelial atypia. A specific complication of hepatic arterial infusion chemotherapy. Cancer 1988;61:663-6.

26. Weidner N, Smith JG, LaVanway JM. Peptic ulceration with marked epithelial atypia following hepatic arterial infusion chemotherapy. A lesion initially misinterpreted as carcinoma. Am J Surg Pathol 1983;7:261-8.

27. Bland KI, Knutson CO, Max MH. Hepatic arterial infusion chemotherapy for cancer of the liver. J Surg Oncol 1980;13: 253-8.

28. Patt YZ, Mavlight GM, Chuang VP, et al. Percutaneous hepatic arterial infusion (HAI) of mitomycin $\mathrm{C}$ and Floxuridine (FUDR): an effective treatment for metastatic colorectal carcinoma in the liver. Cancer 1980;46:261-5.

29. Salomao DR, Mathers WD, Sutphin JE, Cuevas K, Folberg R. Cytologic changes in the conjunctive mimicking malignancy after topical mitomycin C chemotherapy. Ophthalmology 1999;106:1756-60.

30. Holmes GF, Westra WH. Bizarre cytologic atypia mimicking carcinoma in situ (CIS) in sinonasal biopsies following chemotherapy [abstract]. Mod Pathol 1999;12:129A. 